\title{
Design and Realization of DC Motor Speed Measurement and Control Based on an Electromagnetic Sensor
}

\author{
J.Y. Xing \\ Nanjing Communications Institute of Technology \\ Nanjing
}

\begin{abstract}
The design uses the electromagnetic sensor for measuring the speed of DC motor, using 89C52 SCM and Pulse Width Modulation(PWM) software design of the control system for speed control of DC motor for the ratio of air and by the LCD display circuit shows the value of speed, the drive to control the speed of DC motor using $\mathbf{L} 298$ and start and stop. The design is simple and reliable, easy realization, low cost, has important application value for all kinds of new intelligent household appliances.
\end{abstract}

Keywords-electromagnetic sensor; intelligent home appliances; DC motor; circuit design; simulation

\section{INTRODUCTION}

In recent years, the electromagnetic sensor is widely used in all kinds of new household appliances, improves the service quality. This design is the use of electromagnetic sensors to measure and control the speed of DC motor. The permanent magnet in the proper way is fixed on the measured shaft, air gap the electro magnetic sensor placed in the magnet, when the motor rotates, the output voltage of the electromagnetic sensor comprises a speed information, the follow-up processing circuit, we can get the data speed. Produced by the change of electromagnetic sensor pulse field, composed of single chip count, after calculation of the data into the measured speed, then by the LCD display shows the speed of numerical control system software program, design of the DC motor acceleration and deceleration control.

\section{DC MOTOR SPEED MEASUREMENT AND CONT ROL PRINCIPLE}

\section{A. The Theory of Rotating Speed Measurement}

The core element for electromagnetic sensor is Holzer element, it is a sheet made of a semiconductor material and device length, width and height respectively, $1, \mathrm{~B}, \mathrm{D}$. If in the perpendicular to the sheet plane (along the thickness direction of the applied field of B d), plus one in two end a long the $\mathrm{L}$ direction of the electric field, is a current flows through a certain. Because the motion of electrons in a magnetic field, so will be a Lorentz magnetic force, its size:

$$
f=q V B
$$

So that the shift of electron trajectory, respectively to produce electron accumulation or excess charge on two sides

\author{
X.Y. Gu \\ Nanjing Pogue Microelectronics Co \\ Ltd. Nanjing
}

of Holzer components sheet, the formation of Holzer electric field, potential of the two sides of the difference between the Holzer components called Holzer voltage. So that theSoThis makes So the So thatHolzer voltage size:

$$
U_{H}=R_{H} I B / d_{(\mathrm{mV})}
$$

when the magnetic induction intensity when the reverse voltage, Holzer also reverse[1]. Figure.1for the hall element principle structure diagram.

If the control current kept constant, Holzer voltage will vary with the external magnetic field intensity, according to this principle, can be two pieces of permanent magnet rotary edge fixed on a rotating shaft of the motor, rotary table along with the measured shaft to rotate, the magnetic steel will also follow the synchronous rotation, the installation of a Holzer element in the turntable nearby, the turntable rotates along with the shaft when, Holzer element is influenced by the magnetic field generated by the magnet, the output pulse signal. The electromagnetic sensor built-in amplification circuit, the shaping of the signal, the rectangular pulse signal output good. The output frequency and speed is proportional to the measured signal, the pulse frequency or period can be calculated speed.

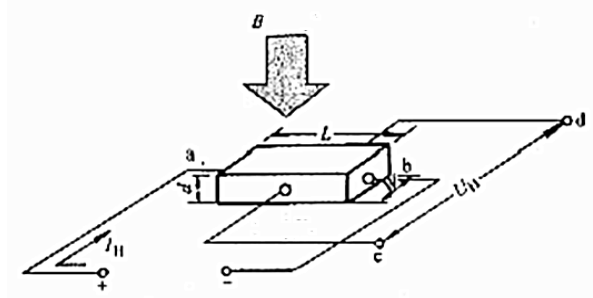

FIGURE I. SCHEMATIC DIAGRAM OF HALL ELEMENT.

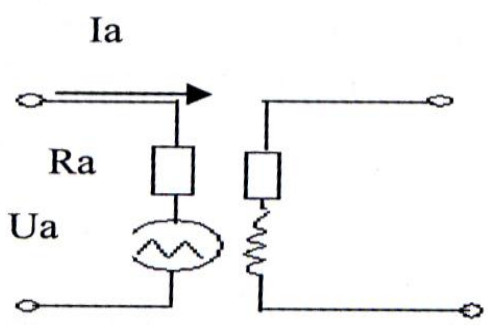

FIGURE II. SCHEMAT IC DIAGRAM OF DC MOTOR. 


\section{B. Speed Control Principle}

The rotation speed of the motor is $n=(\mathrm{Ua}-\mathrm{IaRa})$ with $/ \mathrm{Ca}$, as shown in Figure 2.Ua for the armature voltage, armature current Ia for $\mathrm{Ra}$ for total resistance, armature circuit, $\mathrm{Ca}$ as the motor constant, $\Phi$ for excitation flux. When changing the armature voltage, speed of $\mathrm{N}$ can be changed. The speed of DC motor and is applied to the two ends of the motor voltage size of is proportional to the relationship [2].

The design used in the control of Pulse Width Modulation (PWM) duty cycle to control the speed of DC motor size. The duty ratio is the ratio of connection time and cycle, namely, $\mathrm{M}=\mathrm{t} 1 \mathrm{~T}$. PWM is the DC power supply switching frequency control fixed voltage, changing the voltage across the load, so as to achieve a voltage adjustment process control requirements. The motor when the power is turned on, the motor speed to a maximum of Vmax, if the duty cycle is $M=t 1 / T$, the average speed of the motor is $\mathrm{V}=\mathrm{VmaxM}$. By changing the duty cycle, you can get the motor different average velocity, so as to achieve the purpose of speed.

\section{CIRCUIT DESIGN OF DC MOTOR CONTROL SYSTEM}

\section{A. The Design Block Diagram}

DC motor speed system hardware principle as shown in Figure. 3, with 89C52 as control core, including electromagnetic sensor speed measuring circuit, motor drive circuit, display circuit, keyboard control circuit.

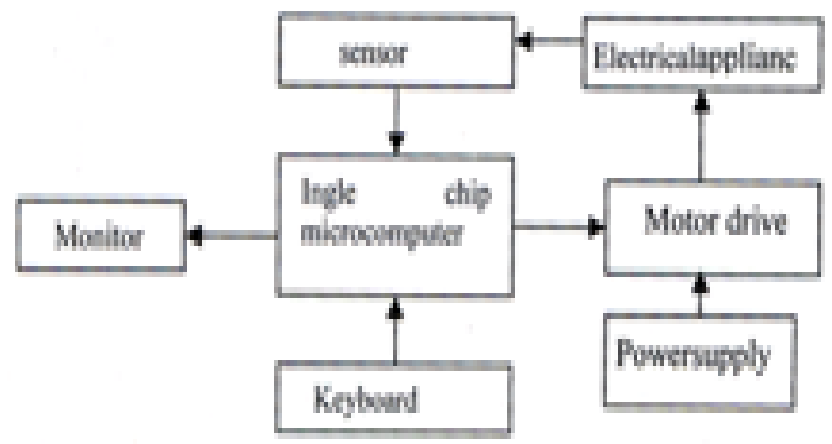

\section{FIGURE III. SPEED CONTROL SYSTEM HARDW ARE BLOCK} DIAGRAM.

\section{B. Speed Measurement Circuit Design}

When the motor rotates, driving the small magnets, Holzer sensorcan be speed signal into a pulse signal output a corresponding frequency, after processing the received signal is output to the counter. The pulse signal frequency and motor speed is proportional to a linear correlation, so the measurement of motor speed, is essentially a measurement of pulse signal of the frequency[3].External connected with the MCU as shown in Figure. 3, when the microcontroller receives external crystal oscillator for low level signals, A3144 also to the SCM input speed data from the detected, chip by programming calculation, we can get the numerical.

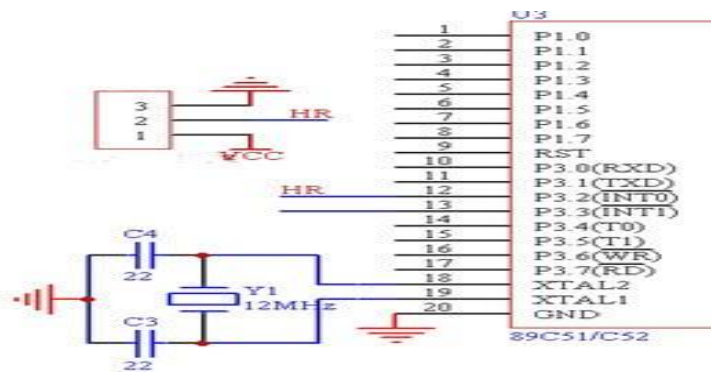

FIGURE IV. THE ELECT ROMAGNETIC SENSOR EXTERNAL CONNECTION DIAGRAM.

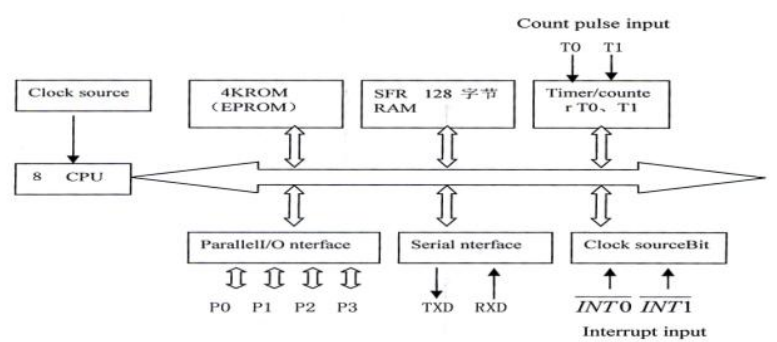

FIGURE V. STC89C52 MICROCONTROLLER ST RUCTURE DIAGRAM.

\section{MCU Circuit Design}

The system uses STC89C52 microcontroller as the core of the rotational speed signal processing. Its basic structure is shown in Figure. 4, the STC89C52 contains 216 bit timer/ counters, $4 \mathrm{~K}$ x 8 bit on-chip FLASH program memory, 48 bit parallel port $\mathrm{I} / \mathrm{O}$. The 16 bit timer / counter is used to realize the frequency measuring signal. A 8 bit parallel port $\mathrm{P} 0, \mathrm{P} 2$ is used to measure the results sent to the display circuit. $4 \mathrm{~K} \times 8$ bit on-chip FLASH program memory is used for placing the system software.

In Figure. 6, the first part is pin 18 and pin 19, they received for an external crystal oscillator module. Selection of crystal for the $12 \mathrm{MHz}$, the capacitor $\mathrm{C} 1$ and $\mathrm{C} 2$ is used to filter the clutter, which stabilized the input STC89C52 single chip waveform. The second part is the reset circuit, pin 9 external reset circuit, when in a low level, circuit without a reset operation, pins 9 through the R1 switch on the power supply of Vcc, this pin 9 is at high potential, STC89C52 microcontroller immediately start the reset procedure itself, start reset.

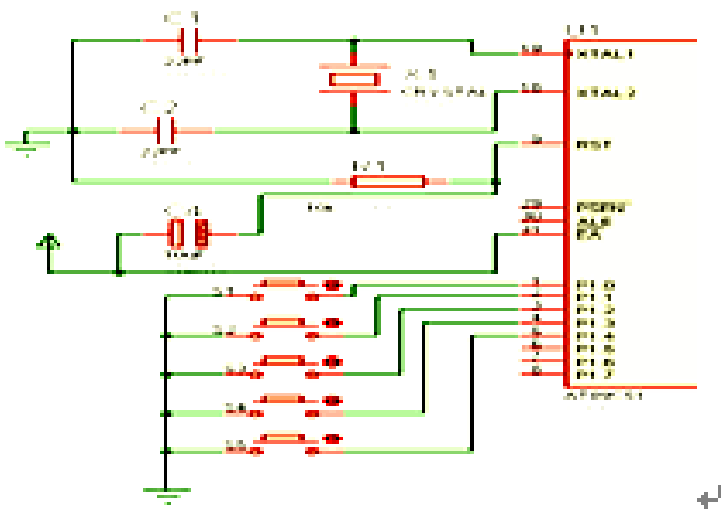

FIGURE VI. THE BASIC CIRCUIT DIAGRAM OF SCM. 


\section{The Design of Motor Drive Circuit}

This design uses L298 DC step motor driver chip, which adopts monolithic integrated plastic installed, is a high voltage, high current whole Shuangqiao drive, controlled by the TTL level standard. L298 logic functions: when L298 enable end is in high level, the IN1 input is PWM signal, the IN2 signal is low, the motor is transferred; the input signal is low, IN2 as the PWM signal, the motor reverse; IN1 and IN2 are the same, the quick stop motor. When the enable input is low, the motor stops rotating.

In the L298 circuit, will enable terminal ENA connected with a single chip computer, make the input acceleration and deceleration of PWM pulse waveformgenerated by the STC89C52 to control DC motor; ENA is directly connected with a power of VCC, can be compiled into to make pin logic INT1 and INT2 logic, with the positive rotation and the reverse rotation to control DC motor. The voltage signal OUT1, OUT2 output to control the motor, the diode is the role of eliminating reverse electro motive force, motor protection circuit, therefore the use of rectifier diode is more appropriate, so by four diodes (D1, D2, D3, D4) control. External connection as shown in Figure 8.

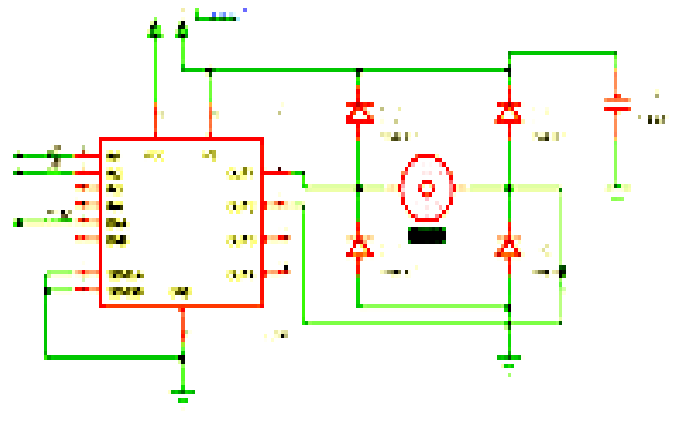

FIGURE VII. L298 EXTERNAL CONNECTION DIAGRAM.

\section{E. LCD Display Circuit Design}

Select LCD1602 as the design of the, which use the physical properties of the liquid, with the voltage on its display control area, there is a voltage will be displayed, which can display graphics. The basic principle of LCD1602 is when the LCD shows a character, because a character consist of a lattice form, only to find the corresponding position on the screen display and memory area, but also to make each byte is divided into different " 1 " and " 0 ", when it is " 1 ", it will be bright, when it is " 0 " not bright. If the controller contains internal character generator, display character is relatively simple, according to the number of columns in the cursor, establish team of liquid crystal display. The design of LCD and the external CPU interface adopts parallel control, communication interface as shown in Figure 8 circuit and single chip microco mputer LCD.

\section{F. Keyboard Input Circuit}

In order to simplify the programming part, is adopted in the design method of lin k serial input: when the key is pressed, the corresponding P1 port directly grounding, because STC89C52 is active low, so the use of MCU internal programming can detect the low potential, can carry out follow-up operation. Among the 5 key functions: starting rotation, respectively $\mathrm{S} 2, \mathrm{~S} 3$ motor stops rotating motor for constant speed rotation, S4 motor, S5 motor deceleration motor of $\mathrm{S} 1$ acceleration. as shown in figure 8 .

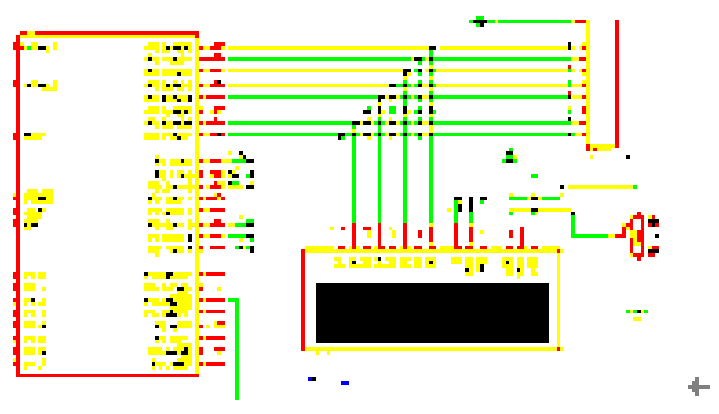

FIGURE VIII. KEYBOARD INPUT CIRCUIT.

\section{DC MOTOR SPEED CONTROL SYSTEM SOFTWARE DESIGN}

\section{A. Design of Detection Program Speed}

Design of electromagnetic sensor A3144 detection rate, and show the use of LCD1602. Before the start of the detection speed, first run the global interrupt enable module, other programs are running in single chip microcomputer to stop. MCU external interrupt by electro magnetic sensor output pulse signal falling edge trigger and counter accumulation and read the A3144 detected data, its calculation, obtain the numerical needs[4]. Finally, through the main function call display, displayed by LCD1602. The flow chart shown in figure 9 .

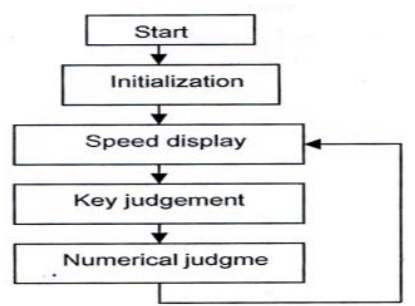

FIGURE IX. SPEED DETECTION PROGRAM FLOW CHART.

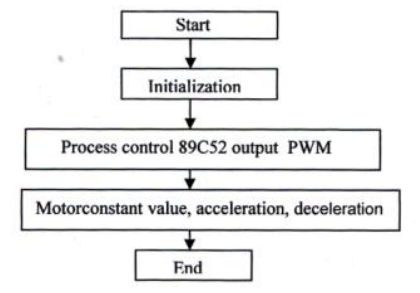

FIGURE X. MOTOR SPEED ADJUST ING FLOW CHART.

\section{B. DC Motor Speed Regulation Program Design}

Using the timer as the timing mode PWM, the resulting output pulse width accurate method using software delay plus to control PWM signal. PWM motor speed control flow chart as shown in figure. 10. Design using the keyboard to set the duty ratio, let the SCM P2.0 port output PWM signal, 
and then through the control of a DC motor drive chip L298 amplification, the input voltage is the voltage at pin two to drive the chip speed difference, a pin of the line is low, another pin produces adjustable speed square wave, so the voltage two the pin of the difference you can control one of the pins to control, so as to realize the acceleration of the motor and reducer [5].

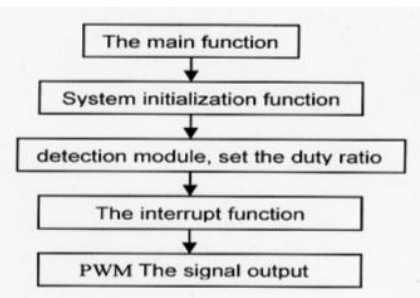

FIGURE XI. PWM WAVEFORM GENERATING FLOW CHART.

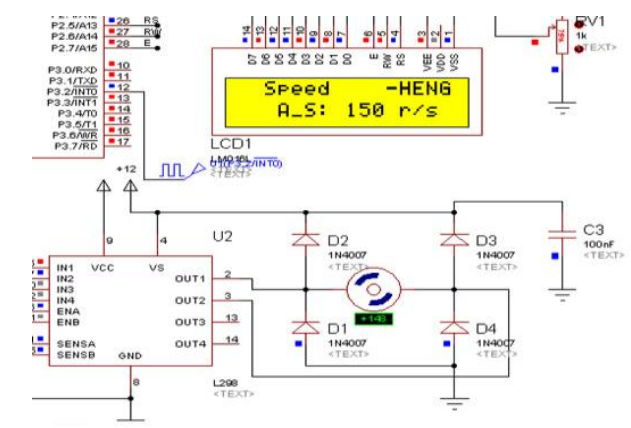

FIGURE XII. DISPLAY THE CURRENT SPEED VALUE.

The design using 89C52 single chip to run the PWM signal generation program, first of all, run the program main function main, and then the parameters of system initialization, including timing / counter set, the definition of 89C52 I/O port, open the T1 interrupt, timer 10us, the initial $30 \%$ duty cycle, the system has 5 main key, start, stop constant speed, acceleration, deceleration. Accelerate button every time duty add $10 \%$, deceleration button every time the duty cycle is reduced by $10 \%$, driving chip to the L298 port of P2.0 single chip output PWM signal, finally using LED real-time display speed value. The program flow chart of as shown in figure 11.

\section{THE EXPERIMENTAL RESULT S OF SIMULATION AND REALITY}

This design through the real and simulation experiment effect is good. As shown in Figure. 13, using PROTEUS and KEIL to simulate and debug the measurement and control system of DC motor, a first project in KEIL under the compiler, running at full speed into debug mode, and then switch to the PRTEUS environment, the screen will display the current velocity and the current state, using the mouse to click the button, the state of the motor by the function key control increase, decrease or stop. In the simulation for continuous rectangular pulse instead of a number of electromagnetic sensor output signal, by adjusting the frequency of the measured signal to reflect how much. The whole measurement system has the advantages of simple structure, low cost, real-time detection of good effect, convenient debugging. The real and simulation results show that, with the single chip microcomputer for DC motor speed control, has a simple hardware circuit, the design procedure is simple and fast operation, easy realization, sensitive control, anti-jamming performance good features.

\section{ACKNOWLEDGEMENT}

Foundation item: Nanjing Communications Institute of Technology 2014research project (project number: JY1408), the host: Xing, Jiang Yong.

\section{REFERENCES}

[1] Lin You, Zhang Jun, Yi fan. Hall sensor signal acquisition and display system design [J]. Modern electronics, in 2009 the first four :191-194.

[2] Xingjiang Yong, Fanze Liang. electrical and electronic technology [M] 2nd edition, Beijing: Science Press, 2010.

[3] Wang Xiangting, Su Xiaolong. Detection system based on the current Hall sensor [J]. Industry and Mine Automation, 20082 :74-176.

[4] Guan Songmin, Zhang Chao, YU Suoping, Chen Qianhong. Following theanalysis of PWM controlled LLC converter and Realization of [J].advanced technology of electrical engineering and energy , 201 1,30 (4) $: 40-45$.

[5] Li Weijun, Han Xiaogang. Zhang Shuai. based microcontroller with soft ware PWM DC motor speed control system [J]. Mechat ronics, 2004, $10(5): 49-51$. 